\title{
Can Heterozygosity of MTHFR and Hyperhomocysteinemia be Risk Factors for Both Retinal/Vitreal Hemorrhages in Retinal Vasculitis and Retinal Vein Occlusion?
}

\author{
Jelena Paovic ${ }^{1}$, Predrag Paovic ${ }^{2}$, Vojislav Sredovic ${ }^{3}$, Zoran Dimcic ${ }^{4}$ \\ ${ }^{1,2}$ University Eye Clinic, Clinical Center of Serbia, Pasterova, Belgrade, Serbia \\ ${ }^{3}$ Primary Health Care Center, Jove Negusevica ,Pecinci, Serbia
}

\author{
${ }^{4}$ Institute for Alergology and Immunology, Clinical Center of Serbia, Pasterova, Belgrade, Serbia \\ Correspondence should be addressed to: Jelena Paovic;
}

Received date: 23 April 2014; Accepted date: 7 July 2014; Published date: 8 June 2015

Academic Editor: Małgorzata Mrugacz

Copyright (c 2015. Jelena Paovic, Predrag Paovic, Vojislav Sredovic, Zoran Dimcic . Distributed under Creative Commons CC-BY 4.0

\begin{abstract}
Retinal vein occlusion (RVO) is one of the most common vascular diseases of the eye and a frequent cause of severe visual loss. It is multifactorial in origin with both local factors and systemic diseases being of etiological importance. Many thrombophilic conditions have recently been identified and studies looking at their potential role in RVO have been undertaken. The aim of this study was to investigate the role of methylenetetrahydrofolate reductase (MTHFR) heterozigosity associated with normal homocysteine level in blood as risk factor for thromboembolic retinal/vitreal manifestations in patients with retinal vasculitis (RV) and RVO where the other risk factors (such as hypertension; atherosclerosis; diabetes; antiphospholipid syndrome) are excluded. Second question was if anticoagulation treatment should be applied together with systemic corticosteroids and/ immunosuppressive therapy? Results obtained from the examined group showed that heterozigosity for MTHFR C677T gene is of statistical significance and correlates with raised values of homocysteine (correlation coefficient $=0.23$ ). At the same time increased levels of homocysteine correlate (correlation coefficient $=0.01$ ) with appearance of RVO. At $r=0.67$, if there is no RV present, existence of a single mutated allele for MTHFR C677T gene does not correlate with RVO. There is however significant correlation between the mutation for MTHFR and hemorrhagic type of RV associated with RVO and/ with isolated hemorrhagic vasculitis without RVO (correlation coefficient $=0.33$; correlation coefficient $=0.27$; respectively).
\end{abstract}

Keywords: retinal vasculitis, retinal vein occlusion, MTHFR C677T polymorphism, homocysteine

Cite this Article as: Jelena Paovic, Predrag Paovic, Vojislav Sredovic, Zoran Dimcic (2015)," Can Heterozygosity of MTHFR and Hyperhomocysteinemia be Risk Factors for Both Retinal/Vitreal Hemorrhages in Retinal Vasculitis and Retinal Vein Occlusion?", Research in Immunology: An International Journal, Vol. 2015 (2015), Article ID 246396, DOI: 10.5171/2015. 246396 


\section{Introduction}

Retinal vasculitis (RV) is an inflammation of retinal blood vessels, which can be isolated or associated with systemic vasculitis. Muffing, narrowing and occlusion of blood vessels lead to retinal ischemia and are basic clinical signs noted on the retina [1]. In absence of retinal ischemia and independently of its etiology, some cases of retinal vasculitis are followed by pronounced retinal and vitreal hemorrhages and/ hemophthalmos. In these cases pathophysiological mechanisms can be based on thrombophilia and/ antiphospholipid syndrome if diabetes, hypertension and atherosclerosis are excluded.

Following diabetic retinopathy, retinal vein occlusion (RVO) is the second most common retinal vascular occlusive disease that can affect people of all ages. According to Hayreh at al. and Mitchel et al. RVO appears to be a condition with a multiple etiology and there is an increased incidence with age (patients are predominantly over 65) $[2,3]$.

RVO may increase venous pressure and lead to retinal capillary decompensation with macular edema and have as consequence decrease/loss of visual efficiency. Exact pathogenesis of RVO is unclear. Primary mechanisms of blood vessel occlusion are degenerative changes of vessel walls, abnormal perivascular changes as well as various hematological factors. Two out of three Virchow's classical factors that play a role in thrombogenesis (stasis, vessel damage and hypercoagulability) have been frequently reported in patients with RVO. Over the past couple of years, especially in case of younger patients, physicians' have been highly interested in thrombophilic risk factors, such as antithrombin deficiency; protein $\mathrm{C}$ and $\mathrm{S}$ deficiency; hyperhomocysteinaemia; factor $\mathrm{V}$ Leiden and II mutations and antiphospholipid antibodies associated with venous thromboembolisms (VTE) [4-7].
Polymorphism (C677T) in methylenetetrahydrofolate reductase (MTHFR) gene has been reported to affect homocysteine levels. In particular, elevated levels of homocysteine are generally accepted to be a risk factor for systemic vascular disease and venous thrombosis. In MTHFR studies, the difference in homocysteine concentration arisen from a single gene mutation is allocated at random $[8,9]$.

Homocysteine is formed in metabolic demethylation process from methionine and it contains a sulphydryl amino group. "Total plasma (or serum) homocysteine" (tHcy) is a combination of free thiol; disulphide-bound to plasma proteins and a combination of homocysteine bound to itself (dimer homocysteine) or with other thiols (bound with cysteine thus forming homocysteine-cysteine mix disulphide) [10]. An abnormal tHcy is defined by an arbitrary cut-off in distribution of concentration found in the "normal population", similarly to hypertension and hypercholesterolemia. Homocysteine is metabolized by remethylation of transsulphuration. One of two methionineconserving remethylation pathways metabolizes homocysteine if protein intake is low. Methionine synthase, $\mathrm{N}^{5}, \mathrm{~N}^{10}$ methylenetetrahydrofolate and $\mathrm{N}^{5}, \mathrm{~N}^{10}$. methylenetetrahydrofolate reductase have an important role in metabolic process of homocysteine in the liver. Similar process occurs in other tissue types. Additionally, vitamin $\mathrm{B}_{12}$ (Cobalamin) is an essential cofactor for methionine synthase [4].

The most common genetic cause of severe hyperhomocysteinemia and classic homocystinuria (congenital homocystinuria) is homozygous deficiency of cystathionine $\beta$-synthase (C $\beta S$ ). Heterozygosity is often associated with normal basal tHcy, and it remains unclear whether heterozigosity is associated with additional risk of vascular events. Homozygous deficiency of MTHFR, both deficiency and impaired activity of methionine synthase, are rare cause of severe hyperhomocysteinaemia [11]. Single 
point mutation in the coding region of MTHFR gene (C-to-T substitution at nucleotide 677) is the most common enzyme defect which occurs with moderately raised tHcy levels. This in turn is associated with thermolabile MTHFR variant which is only half as active [12].

Activated circulating immune complexes against phospholipid membranes and plasma proteins can lead to arterial and venous thromboembolisms. RVO can be one of the first manifestations of antiphospholipid syndrome [1].

\section{Aim}

The aim of this study was to investigate the role of MTHFR heterozigosity associated with normal homocysteine levels in blood as risk factors for thromboembolic retinal/vitreal manifestations in patients with RV (idiopathic or systemic) and RVO where the other risk factors (such as hypertension; atherosclerosis; diabetes; antiphospholipid syndrome) are excluded. Second question was if anticoagulation treatment should be applied together with systemic corticosteroid and/ immunosuppressive therapy (cyclosporine A, metotrexate)?

\section{Methods}

This study consisted of patients who were diagnosed with retinal vascular disorders, between January and June of 2013. All patients underwent full ophthalmological assessment that included: medical history; best corrected visual acuity (BCVA) which was assessed with the use of Snellen's test; slit lamp biomicroscopy; measurement of intraocular pressure (IOP) with Goldmann tonometry; gonioscopy; dilated slit lamp fundus examination with Goldmann 3 mirror lens; and imaging with the Copernicus+ SD-OCT (cross-sectional line at $3 \mu \mathrm{m}$ ) and fluorescein angiography. Complete physical examination was also performed and included measurement of blood pressure; electrocardiogram (ECG); lung and heart examination; as well as abdominal ultrasound. Laboratory tests, which were performed, were: complete blood count; lipid and glucose levels; liver and kidney enzymes; tests for protein C; protein $\mathrm{S}$ and homocysteine. Specific tests for antiphospholipid syndrome such as antinuclear antibodies (ANA and ANA Hep$2)$, anticardiolipin antibodies (aCL), $\beta-2$ glycoprotein 1 antibodies (beta 2GPI), VDRL test, and antithrombin were performed. Genetic tests for MTHFR, Leiden $\mathrm{V}$ and prothrombin 2 were done via polymerase chain reaction (PCR). Ophthalmological and clinical investigations were performed in Uvea Centre, while laboratory tests were conducted at University Clinical Centre in Belgrade.

Taking into consideration possible examiner bias, the following excluding criteria were disorders such as: atherosclerosis, diabetes, hypertension and antiphospholipid syndrome and various ocular manifestations connected with above mentioned disorders. 30 patients were divided into two groups: control group consisted of 20 patients diagnosed with RV without hemorrhagic manifestations on the retina, and examined group of 10 patients with hemorrhagic retinal manifestations with/without RV.

Both quantitative and qualitative statistical analyses were performed (i.e. mean value, standard deviation, correlation). For this single blind research, $\mathrm{P}$ value under 0.05 was considered significant.

\section{Results}

The average age of patients in the control group was 36.3 and \pm 17.4 years and in the examined group it was $33.1 \pm 14.3$, respectively. Systemic retinal vasculitis was present in $19.6 \%$ of patients in the control group, while idiopathic retinal vasculitis was present in $80.4 \%$. Patients in examined group had: retinal vein occlusion (RVO) without retinal vasculitis (40\%), idiopathic retinal vasculitis (IRV) (20\%), and retinal vein branch occlusion (BRVO) with retinal vasculitis (40\%), respectively. Average visual efficiency was 0.7 in the control group and 0.8 in the examined group (Table 1, Figure 1). 
Table 1: Gender, age, visual efficiency and type of retinal vascular disease in control and examined groups

\begin{tabular}{|c|c|c|c|}
\hline & Control group & Examined group & $\mathbf{p}$ \\
\hline Gender (male\% / female \%) & $43.1 / 56.9$ & $30 / 70$ & 0.05 \\
\hline Average age \pm SD & $36.3 \pm 17.4$ & $33.1 \pm 14.3$ & \\
\hline Average visual efficiency & 0.7 & 0.8 & \\
\hline \multirow{2}{*}{\multicolumn{4}{|c|}{\begin{tabular}{|l|l}
\multicolumn{2}{|c|}{ Retinal vascular diseases (\%) } \\
Retinal vasculitis
\end{tabular}}} \\
\hline & & & \\
\hline Systemic & 19.6 & / & \\
\hline Idiopathic & 80.4 & 20 & \\
\hline $\begin{array}{l}\text { Retinal vein occlusion without } \\
\text { vasculitis }\end{array}$ & $\bar{l}$ & 40 & \\
\hline Retinal vein occlusion with vasculitis & 1 & 40 & \\
\hline
\end{tabular}

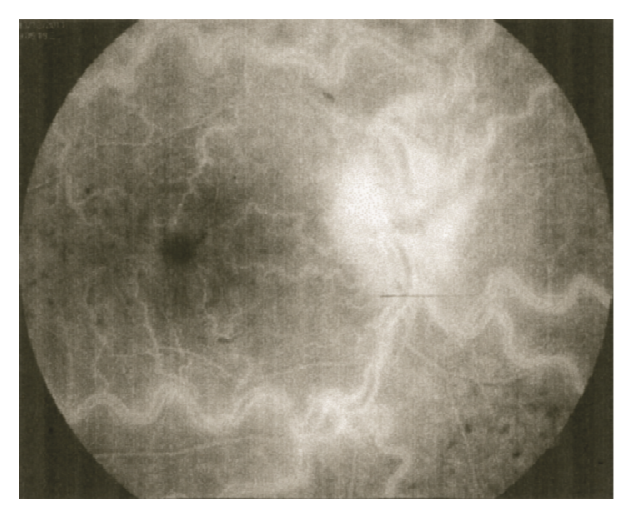

a

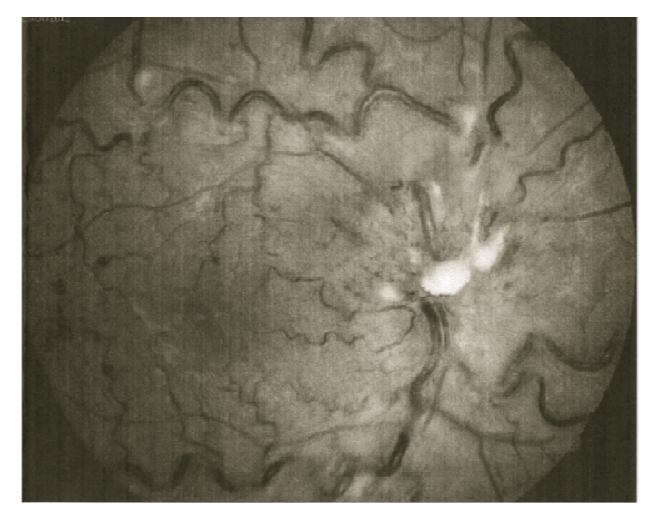

b

Figure 1: Central retinal vein occlusion, fluorescein angiography (a) and photo fundus (b), dilated and tortuous retinal veins, retinal hemorrhage and edema (MTHFR gene mutation and normal level of homocysteine)

Except for one patient with high levels of protein $\mathrm{C}$ and $\mathrm{S}$, the rest of the patients had normal levels. $30 \%$ of patients in examined group had increased values of blood homocysteine, while all patients in the control group had normal values. Control group had no individuals' positive for MTHFR gene mutation (Figure 2). 


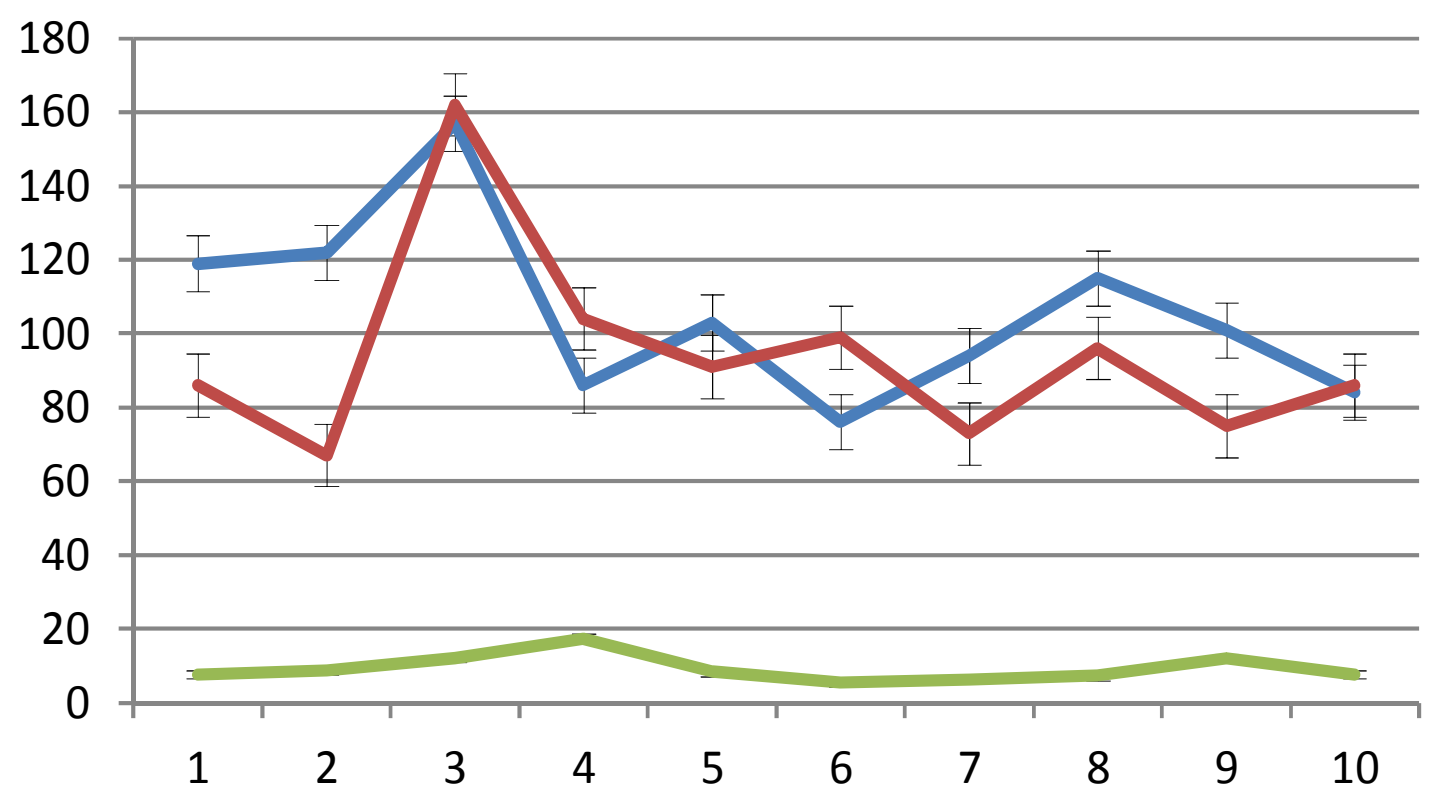

Figure 2: Serum levels of protein $C$, protein $S$ and homocysteine in the examined group

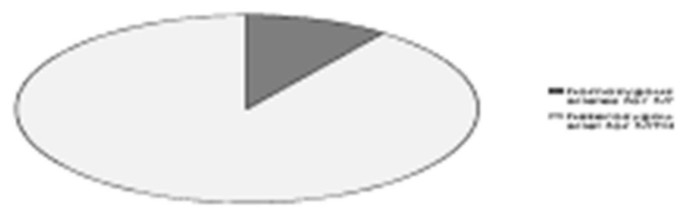

Figure 3: MTHFR C677T gene polymorphism in the examined group

In our sample, results obtained from the examined group showed that occurrence of heterozigosity for MTHFR C677T gene is of statistical significance (Figure 3) and correlates with raised values of homocysteine (correlation coefficient = 0.23). At the same time increased levels of homocysteine correlate (correlation coefficient $=0.01$ ) with appearance of RVO. At $r=0.67$, if there is no RV present, existence of a single mutated allele for MTHFR C677T gene does not correlate with RVO. There is however significant correlation between the mutation for MTHFR and hemorrhagic type of RV associated with RVO and/ with isolated hemorrhagic vasculitis without RVO (correlation coefficient $=0.33$; correlation coefficient $=0.27$; respectively) (Table 2). 
Table 2: Correlation between MTHFR polymorphism, levels of protein $\mathrm{C}$, protein $\mathrm{S}$ and homocysteine, retinal vein occlusion, retinal vasculitis and retinal vasculitis combined with retinal vein occlusion

\begin{tabular}{|l|c|l|l|l|l|l|l|}
\hline & MTHFR & \multicolumn{1}{l|}{ C } & S & tHcy & RVO & RV & RV-RVO \\
\hline MTHFR & 1 & -0.13 & -0.02 & $\mathbf{0 . 2 3}$ & $\mathbf{0 . 6 7}$ & $\mathbf{0 . 2 7}$ & $\mathbf{0 . 3 3}$ \\
\hline C & -0.13 & 1 & 0.44 & 0.24 & 0.65 & -0.53 & -0.21 \\
\hline S & -0.02 & 0.44 & 1 & 0.38 & 0.58 & -0.24 & -0.06 \\
\hline tHcy & $\mathbf{0 . 2 3}$ & 0.24 & 0.38 & 1 & $\mathbf{0 . 0 1}$ & -0.50 & 0.41 \\
\hline RVO & $\mathbf{0 . 6 7}$ & 0.65 & 0.58 & $\mathbf{0 . 0 1}$ & 1 & -0.41 & -0.50 \\
\hline RV & $\mathbf{0 . 2 7}$ & -0.63 & -0.24 & -0.50 & -0.41 & 1 & 0.11 \\
\hline RV-RVO & $\mathbf{0 . 3 3}$ & -0.21 & -0.06 & 0.41 & -0.50 & 0.11 & 1 \\
\hline
\end{tabular}

MTHFR - MTHFR C677T gene polymorphism; $C$ - level of protein C; $S$ level of protein $S$; $t H c y$ - level of total homocysteine; $R V O$ - retinal vein occlusion; $R V$ - retinal vasculitis; $R V-R V O$ - retinal vasculitis combined with retinal vein occlusion
Systemic corticosteroids were used in $80 \%$ of patients in the examined group, while cytostatic therapy was applied in $30 \%$ of them. Out of $40 \%$ of patients treated by Nadroparin calcium injections only one had RV. All patients underwent acetylsalicylic acid treatment (Figure 4).

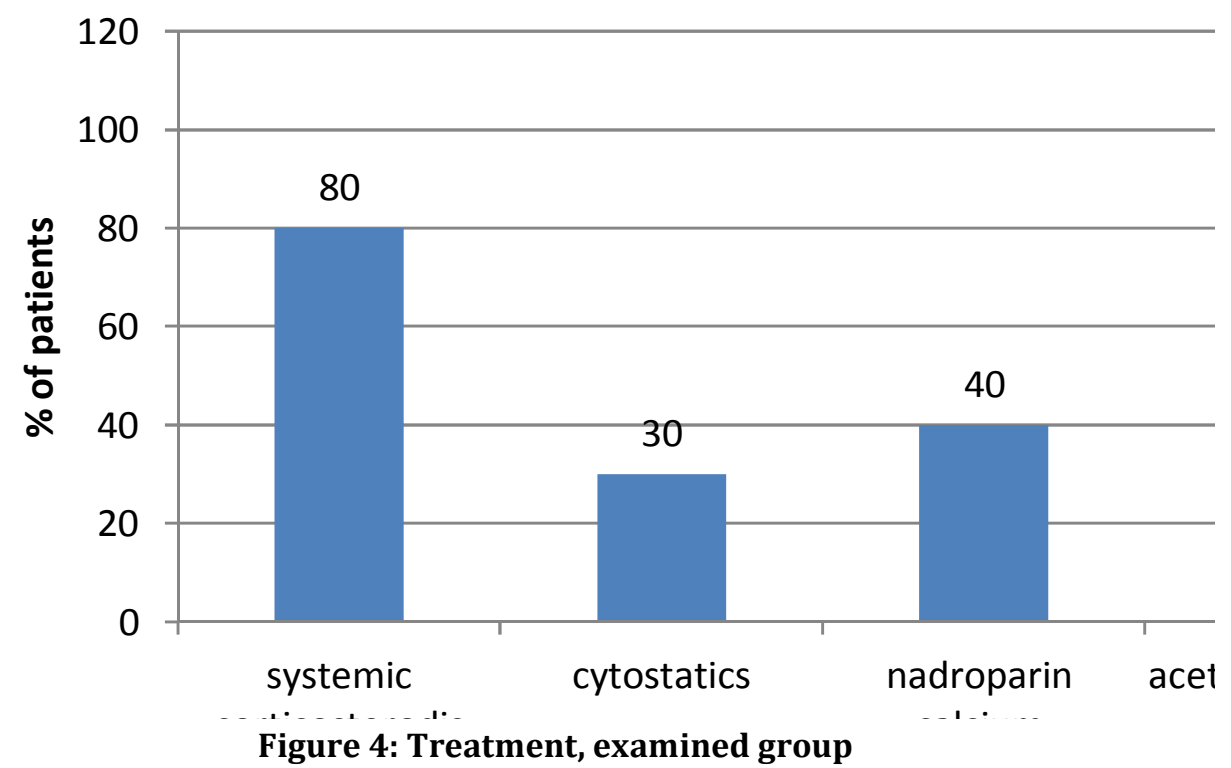

Thrombophilic manifestations of $\mathrm{RV}$ in patients heterozygous for MTHFR gene mutation were one of the reasons for anticoagulant therapy in treatment of two patients with severe retinal and vitreal hemorrhages. Parallel to the previously 
mentioned anticoagulant therapy, these patents also received corticosteroid and immunosuppressive agents (Figure 5).

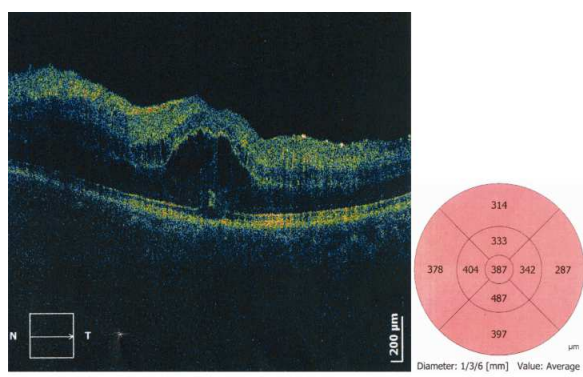

a1

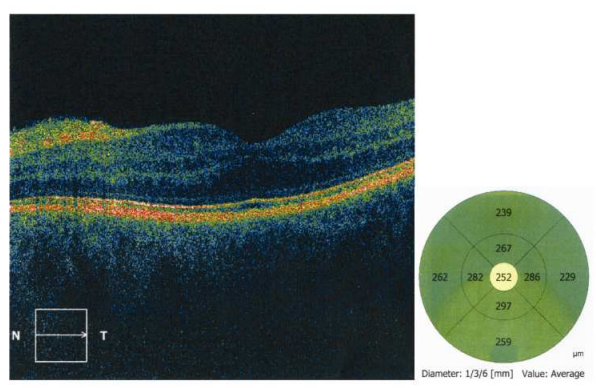

a2
Following treatment, retinal hemorrhages and edema were reduced and macular edema disappeared all together.

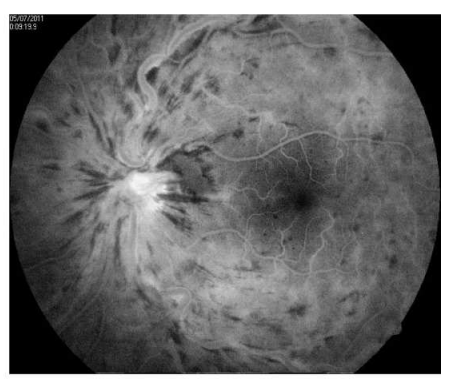

b1

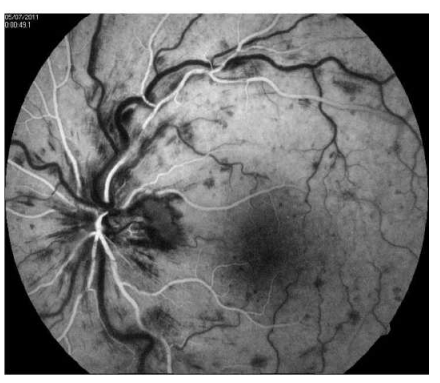

b2

Figure 5: Retinal vein occlusion follow up patient which was treated with anticoagulant and corticosteroid therapy (a - macular edema, optical coherent tomography, b angiography, 1 - initial examination, 2 - follow up)

\section{Discussion}

Retinal vein occlusion (RVO) is one of the most common vascular diseases of the eye and a frequent cause of severe visual loss. Numerous risk factors including arterial hypertension, diabetes mellitus, and arteriosclerosis have been identified. It is multifactorial in origin with both local factors and systemic diseases being of etiological importance. Many thrombophilic conditions have recently been identified, and studies looking at their potential role in RVO have been undertaken.

Gene polymorphisms affecting hemostasis may also play a role in pathogenesis of RVO and studies to determine prevalence of genetic polymorphisms of various factors implicated in hypercoagulability among patients with RVO have been performed [13].
Thrombophilia plays an important role in the pathogenesis of RVO in patients who have no acquired risk factors. Screening for thrombophilia in patients where these factors have been excluded, seem to be somewhat controversial in that they leave a question concerning the association of RVO and hereditary thrombophilial abnormalities unanswered. Various coagulation disorders induced by genetic mutations (antithrombin III gene mutations, protein $\mathrm{C}$ and protein $\mathrm{S}$ deficiencies, factor $\mathrm{V}$ Leiden mutation, or MTHFR mutations) are often associated with an increased risk for both systemic and retinal vein occlusion [14-21].

In the year 1976, Wilcken presented a first case controlled study which suggested that the higher than normal levels of homocysteine which can otherwise be found in a "normal" population seem to be a predisposing factor for RVO. Some studies which have been performed at a later date 
have also confirmed it to be a risk factor for venous thrombosis [22, 23, 24]. On the other hand, our results show that RVO and hemorrhagic retinal manifestations can occur in patients with retinal vasculitis at normal levels of homocysteine.

Although the exact mechanisms by which hyperhomocysteinaemia causes thrombosis are unknown, it has been shown to be toxic to endothelial cells, to impair thrombomodulin expression and to reduce protein $\mathrm{C}$ activation via vascular endothelial cell activator [25-28].

However, a mild form of hyperhomocysteinaemia was identified due to a thermolabile variant of MTHFR [29, 30].

There was some contradictory evidence concerning homozygosity and heterozigosity of MTHFR C677T gene and its mutations. Some results indicated that elevated levels of total homocysteine (tHcy) were associated with RVO, but not with homozygosity for the MTHFR C677T gene [31]. In case of homozygotes' prevalence for MTHFR C677T gene mutation and subsequently for RVO is significantly higher than that of heterozygotes' [32]. Our results obtained from the examined group showed that heterozigosity for MTHFR C677T gene are of statistical significance and correlate with raised values of homocysteine. At the same time increased levels of homocysteine correlate with appearance of RVO. If there is no $\mathrm{RV}$ present, the existence of a single mutated allele for MTHFR C677T gene does not correlate with RVO. There is however a significant correlation between the mutation for MTHFR and hemorrhagic type of RV associated with RVO and/ with isolated hemorrhagic vasculitis without RVO; respectively.

There are many causes for hyperhomocysteinaemia including certain vitamin deficiencies (pyridoxine, folic acid, vitamin $\mathrm{B}_{12}$ ), chronic illnesses (chronic renal failure, diabetes, hypertension, cancer), drugs (metotrexate, anti-epileptic agents), and various rare enzyme deficiencies (some of which have been mentioned above). It appears however that many "normal" patients with MTHFR genotype have normal homocysteine levels (phenotypic expression is critically dependent on both folate levels and on folic acid intake) [33].

There exists a high association between the MTHFR gene C677T mutation and Behcet's disease, and a mutation of MTHFR gene associated with an increased risk of ocular involvement suggesting that genetic instability may be caused by homocysteine lowering therapy [34]. According to clinical features for Behcet's' disease, stratification analyses did not reveal any other association except for the one for the response to colchicine that was shown to be influenced by MTHFR C677T mutation [35].

Bearing in mind that patients with RV associated with retinal and vitreal hemorrhage in absence of hyperhomocysteinemia have been under cytostatic and corticosteroid therapy, heterozygosity for MTHFR gene mutation may be related to other risk factors (i.e. chronicity of disease; vitamin deficiencies and cytostatic drugs). Patients in the control group with RV, but without retinal and vitreal hemorrhage, had normal homocysteine values and no MTHFR mutation.

Keeping with other thrombophilic conditions, other hereditary and acquired pro-thrombotic disorders are in synergy with each other and hyperhomocysteinaemia. [36, 37].

\section{Acknowledgment}

The authors of this manuscript would like to give special acknowledgment to prof. $\mathrm{dr}$ Anka Stanojević - Paović, the chief medical officer at Uvea Center, for scientific and professional help and support that she has provided during the research process.

\section{References}

1. Stanojevic-Paovic, A., Paovic, J. and Paovic, P. (2008) Uveitisi, School of Medicine, University of Belgrade, Belgrade, Serbia. 
2. Hayreh, S. S., Zimmerman, M. B. and Podhajsky, P. (1994) "Incidence of various types of retinal vein occlusion and their recurrence and demographic characteristics," American Journal of Ophthalmology, 117 (4) 429-441.

3. Mitchel, P., Smith, W. and Chang, A. (1996) "Prevalence and associations of retinal vein occlusion in Australia. The Blue Eye Mountain Study," Archives of Ophthalmology, 114 (10) 1243-1247.

4. Hankey, G. J. and Eikelboom, J. W. (1999) "Homocysteine and vascular disease," Lancet, 354 (9176) 407-413.

5. Fegan, C. D. (2002) "Central retinal vein occlusion and thrombophilia," Eye, 16 (1) 98-106.

6. Pinna, A., Carru, C., Zinellu, A., Dore, S., Deiana, L. and Carta, F. (2006) "Plasma homocysteine and cysteine levels in retinal vein occlusion," Investigative Ophthalmology \& Visual Science, 47 (9) 4067-4071.

7. Beaumont, P. E. and Kang, H. K. (2002) "Clinical characteristics of retinal venous occlusions occurring at different sites," British Journal of Ophthalmology, 86 (5) 572-580.

8. Wald, D. S., Law, M. and Morris, J. K. (2002) "Homocysteine and cardiovascular disease: evidence on causality from a metaanalysis," British Medical Journal, 325 (7374) 1202.

9. Den Heijer, M. (2003) "Hyperhomocysteinaemia as a risk factor for venous thrombosis: an update of the current evidence," Clinical Chemistry and Laboratory Medicine, 41 (11) 1404-1407.

10.Ueland, P. M. (1995) "Homocystein species as components of plasma redox thiol status," Clinical Chemistry, 41 (3) 340342.

11.Ogier de Baulny, H., Gerard, M., Saudubray, J. M. and Zittoun, J. (1998) "Remethylation defects: guidelines for clinical diagnosis and treatment," European Journal of Pediatrics, 157 (Suppl 2) S77-83.

12.Kang, S. S., Zhou, J., Wong, P. W., Kowalisyn, J. and Strokosch, G. (1988) "Intermediate homocysteinemia; a thermolabile variant of methylenetetrahydrofolate reductase," American Journal of Human Genetics, 43 (4) 414-421.

13.Weger, M., Renner, W., Steinbrugger, I. and all. (2005) "Role of thrombophilic gene polymorphisms in branch retinal vein occlusion," Ophthalmology, 112 (11) 19101915.

14.Incoravaia, C., Lamberti, G., Parmeggiani, F. and all. (1999) "Idiopathic central vein occlusion in a thrombophilic patient with the heterozygous 20210 G/A prothrombin genotype," American Journal of Ophthalmology, 128 (2) 247-248.

15.Rehak, M., Krcova, V., Slavik, L. and all. (2010) "The role of thrombophilia in patients with retinal vein occlusion and no systemic risk factors," Canadian Journal of Ophthalmology, 45 (2) 171-175.

16.Biancardi, A. L., Gadelha, T., Borges, W. I. and all. (2007) "Thrombophilic mutations and risk of retinal vein occlusion," Arquivos Brasileiros de Oftalmologia, 70 (6) 971-974.

17.Dahlback, B., Carlsson, M. and Svensson, P. J. (1993) "Familial thrombophilia due to a previously unrecognised mechanism characterized by a poor anticoagulant response to activated protein C," Proceedings of the National Academy of Science of the United States of America, 90 (3) 1004-1008.

18.Koeleman, B. P. C., Reitsma, P. H., Altaart, C. F. and Bertina, R. M. (1994) "Activated protein $\mathrm{C}$ as an additional risk factor for thrombosis in protein C-deficient families," Blood, 84 (4) 1031-1035.

19.Koeleman, B. P. C., Van Rumpt, D., Hamulyak, K., Reitsma, P. H. and Bertina, R. M. (1995) "Factor V Leiden: an additional risk factor for thrombosis in protein $S$ 
deficient families?," Thrombosis and Haemostasis, 74 (2) 580-583.

20.Incoravia, C., Parmeggiani, F., Costagliola, C., Lamberti, G., Ferraresi, P., Bernardi, F. and Sebastiani, A. (2001) "The heterozygous $20210 \mathrm{G} / \mathrm{A}$ genotype prevalence in patients affected by central and branch retinal vein occlusion," Graefe's Archive for Clinical and Experimental Ophthalmology, 239 (4) 251-256.

21.Rehak, M., Rehak, J., Muller, M. and all. (2008) "The prevalence of activated protein $\mathrm{C}$ (APC) resistance and factor $\mathrm{V}$ Leiden is significantly higher in patients with retinal vein occlusion without general risk factors. Case-control study and metaanalysis," Thrombosis and Haemostasis, 99 (5) 925-929.

22.Wilcken, D. E. and Wilcken, B. (1976) "The pathogenesis of coronary artery disease. A possible role for methionine metabolism," The Journal of Clinical Investigation, 57 (4) 1079-1082.

23. Den Heijer, M., Rosendaal, F. R., Blom, H. J., Gerrits, W. B. and Bos, G. M. (1998) "Hyperhomocysteinaemia and venous thrombosis: a meta-analysis," Thrombosis and Haemostasis, 80 (6) 874-877.

24.Mudd, S. H., Skovby, F., Levy, H. L. and all. (1985) "The natural history of homocystinuria due to cystathionine betasynthase deficiency," American Journal of Human Genetics, 37 (1) 1-31.

25.Harker, L. A., Slichter, S. J., Scott, C. R. and Ross, R. (1974) "Homocystinemia, vascular injury and arterial thrombosis," New England Journal of Medicine 291 (11) 537-543.

26. Rodgers, G. M. and Kane, W. H. (1986) "Activation of endogenous factor $\mathrm{V}$ by a homocysteine-induced vascular endothelial cell activator," Journal of Clinical Investigation, 77 (6) 1909-1916.

27.Rodgers, G. M. and Conn, M. T. (1990) "Homocysteine, an atherogenic stimulus, reduces protein $\mathrm{C}$ activation by arterial and venous endothelial cells," Blood, 75 (4) 895-901.
28.Lentz, S. R. and Sadler, J. E. (1993) "Homocysteine inhibits von Willebrand factor processing and secretion by preventing transport from the endoplasmic reticulum," Blood, 81 (3) 683-689.

29.Kang, S. S., Zhou, J., Wong, P. W., Kowalisyn, J. and Strokosch, G. (1988) "Intermediate homocysteinemia: a thermolabile variant of methylenetetrahydrofolate reductase," American Journal of Human Genetics, 43 (4) 414-421.

30.Frosst, P., Blom, H. J., Milos, R. and all. (1995) "A candidate genetic risk factor for vascular disease: a common mutation in methylenetetrahydrofolate reductase," Nature Genetics, 10 (1) 111-113.

31.McGimpsey, S. J., Woodside, J. V., Cardwell, C., Cahill, M. and Chakravarthy, U. (2009) "Homocysteine, methylenetetrahydrofolate reductase C677T polymorphism, and risk of retinal vein occlusion: a meta-analyses," Ophthalmology, 116 (9) 1778-1787.

32.Marcucci, R., Giusti, B., Betti, I. and all. (2003) "Genetic determinants of fasting and post-methionine hyperhomocysteinemia in patients with retinal vein occlusion," Thrombosis Research, 110 (1) 7-12.

33.Jacques, P. F., Bostom, A. G., Williams, R. R. and all. (1996) "Relation between folate status, a common mutation in methylenetetrahydrofolate reductase, and plasma homocysteine concentrations," Circulation, 93 (1) 7-9.

34.Ozkul, Y., Evereklioglu, C., Borlu, M. and all. (2005) "5,10Methylenetetrahydrofolate reductase C677T gene polymorphism in Behcet's patients with or without ocular involvement," The British Journal of Ophthalmology, 89 (12) 1634-1637.

35. Karakus, N., Yigit, S., Kalkan, G. and all. (2012) "Association between the methylene tetrahydrofolate reductase gene C677T and colchicine unresponsiveness in 
Behcet's disease," Molecular Vision, 18 1696-1700.

36.Graham, I. M., Daly, L. E., Refsum, H. M. and all. (1997) "Plasma homocysteine as a risk factor for vascular disease. The European Concerted Action Project," Jama: The Journal of the American Medical Association, 277 (22) 1775-1781.
37.De Stefano, V., Zappacosta, B., Persichilli, S. and all. (1999) "Prevalence of mild hyperhomocysteinaemia and association with thrombophilic genotypes (factor V Leiden and prothrombin G20210A) in Italian patients with venous thromboembolic disease," British Journal of Haematology, 106 (2) 564-568. 